\title{
LA REPUTACIÓN ONLINE Y SU IMPACTO EN LA POLÍTICA DE PRECIOS DE LOS HOTELES
}

\author{
Patricia Diana-Jens \\ Antonio Rodríguez Ruibal \\ Universidad a Distancia de Madrid
}

\section{RESUMEN}

En este trabajo se demuestra que una buena reputación online, entendiendo por ello la primera posición de un determinado destino según el índice de popularidad de Tripadvisor, permite a los hoteles que ostentan dicho posicionamiento ofrecer precios de venta más altos que los de sus competidores. En concreto, un establecimiento situado en la primera posición de un destino, aparte de tener mayor visibilidad y por lo tanto posibilidades de compra por parte del consumidor, establece de media sus precios un $22,26 \%$ más altos que los precios de su set de competidores para un mismo tipo de producto.

Palabras clave: Reputación online; social media; revenue management; Tripadvisor; precios.

\section{Online reputation and its impact on hotel pricing strategies}

\section{ABSTRACT}

In this study it is proven that a good online reputation, understood as the first position in a geographical area following the Tripadvisor's Popularity Index, allows hotels holding this first position to offer higher prices than the ones offered by their competitors. Specifically, an hotel holding the first position, apart from having more visibility and therefore more choices of being booked by the consumer, offers its rates an average of $22,26 \%$ higher than the rates offered by the competitive set for the same type of product.

Keywords: Online reputation; social media; revenue management; Tripadvisor; pricing strategy.

Recibido: 14 de julio de 2014

Devuelto para su revisión: 19 de noviembre de 2014

Aceptado: 6 de febrero de 2015

Universidad a Distancia de Madrid. UDIMA. Carretera de la Coruña Km. 38,500. Vía de servicio no 15 28400 Collado Villalba. MADRID (España).E-mail: patricia.diana@udima.es, antonio.rodriguez@udima.es 


\section{INTRODUCCIÓN}

La web 2.0 ha supuesto un cambio de paradigma en el sector hotelero en los últimos años. Por una parte los clientes tienen todos los medios para obtener la información que necesitan en el momento de decidir la compra, gracias a la irrupción de los nuevos canales de comunicación conocidos como Social Media. Tripadvisor, Facebook, Youtube son sólo algunos de los ejemplos de los medios existentes hoy en día para que un cliente pueda acceder a información que no va a encontrar en la página web del hotel.

Por otra parte, los clientes pueden aportar sus opiniones, fotos, vídeos, puntuaciones, etc. en diversos medios de Internet tras una experiencia de alojamiento en un hotel.

Además, los hoteleros tienen a su disposición una información ingente que antes era imposible de obtener: información sobre lo que opinan los clientes de su hotel, información sobre lo que opinan los clientes de sus competidores, precios y productos ofertados por la competencia...

Aparte de disponer de esa información, el hotelero puede comunicarse con su cliente a través de estos nuevos canales de Social Media de manera directa y pública, lo cual refuerza la vinculación del establecimiento con su cliente, independientemente de la naturaleza positiva o negativa de dicha comunicación.

Dicho cambio de paradigma ha supuesto y supone un enorme dolor de cabeza para los hoteleros, que desbordados por la velocidad de los cambios que estamos viviendo, no son capaces de aprovechar toda esa información para mejorar sus resultados empresariales.

En primer lugar tienen que digerir ese importante flujo de información proveniente de diversos canales mediante herramientas que permitan obtener fácilmente dicha información en un formato útil y sencillo. Hoy en día existen diferentes empresas que comercializan este tipo de herramientas enfocadas al sector hotelero, pero todavía son pocos los hoteleros que deciden invertir en ellas.

$\mathrm{Y}$ en segundo lugar tienen que poder utilizar dicha información para su provecho. Por una parte para incrementar y mejorar el contacto directo con el cliente, para mejorar su producto e instalaciones, pero por otra parte, y es en la que nos centramos en este trabajo, para poder mejorar los resultados empresariales.

La importancia de dicha información es tal que debería influir como una variable más en la estrategia de marketing del hotel, tanto en el posicionamiento en un mercado como en su política de precios.

A lo largo de este trabajo intentaremos demostrar la influencia que tiene la reputación online, entendida como el conjunto de opiniones creadas a través de los diferentes canales de Social Media, en los resultados de un hotel.

Más en concreto, nos centraremos en el estudio de la influencia que tiene el Índice de Popularidad de Tripadvisor en la política de precios de los hoteles. Dicho índice está basado en las opiniones de los usuarios y refleja el posicionamiento de un hotel con respecto a los hoteles similares situados en la misma zona geográfica.

Tripadvisor es una página web donde se recogen los comentarios y opiniones de viajeros de todo el mundo (en el momento del estudio, Tripadvisor recoge más de 150 millones de opiniones) y se ha convertido en la página de comentarios líder en el mundo (Market Metrix, 2013, además de la principal fuente de reputación online en el sector hotelero. 
Los objetivos principales de este trabajo son dos. En primer lugar, comprobar si existe relación entre el posicionamiento en el Ranking de Tripadvisor y el precio ofrecido por el hotel. Para ello se analiza si los hoteles situados en la primera posición de su categoría y en su zona geográfica según el Ranking de Tripadvisor ofrecen precios más altos que los hoteles competidores. En segundo lugar, proponer la reputación online como variable a tener en cuenta por parte de un hotel a la hora de establecer sus precios en relación con su competencia

Este trabajo se ha realizado en dos fases diferenciadas. En la primera fase se ha revisado la literatura referente al impacto de la reputación online en las políticas de precios de los hoteles. Para ello se empieza revisando los conceptos de reputación corporativa y reputación online, pasando después a estudiar el impacto de la reputación online en los resultados de una empresa. Una vez establecido este marco, centramos el tema ya en el sector hotelero, analizando la influencia de la reputación online en los resultados de un hotel y más en concreto, en la política de precios de un hotel, parte integrante del Revenue Management ${ }^{1}$ como forma de gestión de un establecimiento hotelero. En la segunda fase se realiza un trabajo de investigación para poder sacar conclusiones empíricas que nos acerquen a los objetivos de este trabajo.

\section{REVISIÓN DE LA LITERATURA}

No existe un concepto unánime ni una definición clara de reputación corporativa, puesto que la reputación corporativa se puede estudiar desde varios puntos de vista.

La reputación se puede entender (Del Fresno, 2012, pp. 13-14) «como la construcción social alrededor de la credibilidad, fiabilidad, moralidad y coherencia que se tiene de una persona, ente, organismo, institución, empresa, etc. Por ello sólo es parcialmente controlable por el sujeto ya que se crea y recrea a partir de las percepciones que conforman un estado de opinión, consideración y valoración de otros (...) Asimismo, la reputación es una construcción social, un producto creado y acumulado de forma colectiva y que de manera inevitable tiene efectos positivos o negativos sobre el despliegue social, económico, etc. al tener una evidente connotación pública».

De dicha definición podemos destacar algunas características clave:

- la reputación se crea a partir de percepciones

- la reputación sólo es parcialmente controlable por parte de la empresa

- la reputación se crea y acumula de forma colectiva

- la reputación tiene efectos en las empresas, positivos o negativos

El vínculo entre reputación corporativa y resultados empresariales ha sido objeto de estudio desde diferentes puntos de vista: el financiero, el de marketing, el punto de vista sociológico y el estratégico.

1 Revenue Management: forma de gestión de un establecimiento hotelero, que consiste en vender el producto adecuado, al precio adecuado, en el momento adecuado, al cliente adecuado en el canal de distribución adecuado. 
Una buena reputación tiene varios efectos en las empresas (Chun, 2005) (Hernández Estárico, Fuentes Medina, \& Morini Marrero, 2012):

- Mayor satisfacción del cliente

- Incremento de las ventas

- Lealtad del cliente

- Incremento de beneficios

- Satisfacción de los empleados

- Mayor retención del personal

- Capacidad de atracción de personal mejor preparado

A lo largo de este trabajo nos centraremos principalmente en el impacto que dicha reputación tiene en el aspecto económico.

En los últimos años, la reputación corporativa ha sufrido un cambio innegable debido principalmente al desarrollo de las nuevas tecnologías e Internet.

La reputación online se puede entender (Del Fresno, 2012, p. 14) como «el resultado de lo que los clientes, ex-clientes, futuros clientes, empleados, etc. dicen, escriben y transmiten a otros en cualquier parte de los medios sociales de internet o social media a partir de sus percepciones y experiencia en cualquier momento de su relación, directa o indirecta, con esa marca».

Es por ello que hoy en día la gestión adecuada de la reputación online de una empresa, entendida como el conjunto de opiniones que se vierten en Internet sobre dicha empresa, sus marcas, bienes o servicios, resulta en la actualidad un aspecto vital para la competitividad empresarial (Hernández Estárico, Fuentes Medina, \& Morini Marrero, 2012).

Algunas de las características más importantes de la reputación online, en contraposición con la idea de reputación tradicional, son (Hernández Estárico, Fuentes Medina, \& Morini Marrero, 2012):

- la variable prioritaria en la reputación online es la relativa a la calidad de los productos y/o servicios

- la información sobre una empresa difundida en la web es muy perdurable, puesto que se almacena en servidores no controlables por la empresa

- la información generada sobre una empresa es fácilmente localizable por los buscadores

- la información que circula en internet sobre una empresa es muy rica y variada, puesto que proviene de fuentes diversas, es cada vez más colaborativa y depende en mayor medida de la opinión de terceros

- la información fluye muy rápidamente a través de internet, con las consecuencias que ello conlleva para una adecuada gestión de la reputación.

En otro orden de cosas, el término Social Media provoca bastante confusión entre los investigadores y es conveniente resaltar su diferencia con los términos Web 2.0 y User Generated Content, utilizados en la literatura como similares. Según Kaplan et al., (2010) la web 2.0 sería la plataforma tecnológica para la evolución del Social Media, cuyo conte- 
nido se crea y modifica continuamente por todos los usuarios de una manera colaborativa. Por $\mathrm{UGC}^{2}$ se entiende, siempre según los mismos autores, el diverso contenido creado y compartido por los usuarios de Social Media. Así, Social Media se puede definir como un grupo de aplicaciones basadas en internet que se basan en la web 2.0 y que permiten la creación e intercambio del Contenido Generado por los Usuarios.

En este sentido, el auge del Social Media, y más en concreto de los portales de internet donde los usuarios comentan su experiencia o valoran el producto o servicio adquirido ha cambiado de manera sustancial el concepto de reputación de las empresas.

No es mucha la literatura científica que estudia el impacto de la reputación online en los resultados económicos de la empresa, puesto que la mayoría de los artículos se centran en el impacto que tiene dicha reputación en la decisión de compra del consumidor. Hemos podido sin embargo analizar las principales aportaciones sobre el tema.

Lynch y Ariely (2000) estudian el impacto de la transparencia en la búsqueda de productos en los precios, concluyendo que en el caso de productos diferenciados como el vino (objeto de su investigación), reducir el coste de la búsqueda (o lo que es lo mismo a sus efectos, aumentar la transparencia y la información al consumidor en Internet) reduce la sensibilidad al precio en el cliente. Sugieren por lo tanto que los productores permitan la máxima transparencia en la información de compra, al tiempo que una mayor diferenciación en su producto que evite una competencia basada únicamente en el precio.

Resnick et al. (2006) estudia el impacto que tiene la reputación de un vendedor en EBay $^{3}$ en la predisposición al pago de los compradores. Mediante su investigación demuestra que el mercado recompensa (es decir, está dispuesto a pagar más) al vendedor de Ebay que ha acumulado mucho feedback positivo: los compradores están dispuestos a pagar a un vendedor con fuerte reputación un $8,1 \%$ más de media que a un vendedor nuevo.

Chevalier y Mayzlin (2006) estudian el impacto de las opiniones online referidas a los libros disponibles en Amazon y en Barnes \& Noble, concluyendo que si un libro en particular tiene más opiniones y opiniones mejor valoradas en una de las dos webs, dicho libro incrementará su cuota de mercado de ventas en dicha web.

Graham y Bansal (2007) realizan un estudio para investigar la predisposición al pago de los usuarios de líneas aéreas. Su investigación demuestra que los consumidores valoran la buena reputación entre las líneas aéreas y que además están dispuestos a pagar más por una mejor reputación. Y ello porque una buena reputación sugiere que los productos y servicios de la empresa tienen una calidad mayor, además de suponer que la empresa es responsable y trata bien a sus clientes. Concretamente, los resultados de su estudio indican que por cada punto de incremento en la reputación de la línea aérea, los clientes están dispuestos a pagar \$18 más por un billete de avión. El hecho de que la reputación corporativa tenga un peso importante en la relación entre características de la empresa y predisposición del cliente a pagar tiene implicaciones a nivel estratégico, y sugiere que las empresas que quieran incrementar la facturación tendrán que elaborar un buen plan de gestión de la reputación corporativa.

2 UGC: User Generated Content - contenido generado por el usuario.

3 Ebay: portal de subastas para compraventa entre particulares en internet. 
En el ámbito de las películas de cine, Dellarocas et al. (2007) estudia el valor de las puntuaciones online de las películas a la hora de predecir la facturación de dichas películas. Su investigación concluye que las opiniones online de las películas constituyen una prueba medible de la información boca-oreja que debería ser explotada por las productoras de las películas para prever y planificar la facturación.

Desde otro punto de vista, Feng Zhu et al. (2010) estudia el impacto de las opiniones online en las ventas de los videojuegos, con dos conclusiones interesantes. Por una parte, las opiniones online tienen un influencia mayor en los jugadores con más experiencia en Internet. Por otra parte, las opiniones online tienen una mayor influencia en las ventas de los juegos menos populares que en los más conocidos, aludiendo en su investigación al teorema de Long Tail de Anderson. Sugiere por lo tanto que los productores que se mueven en nichos de mercado y aquellos que venden online deberían tener una estrategia definida para la gestión de las opiniones online, puesto que pueden afectar a sus ventas de una manera significativa.

De Maeyer (2012) hace una revisión del estado de la cuestión en cuanto al impacto de las opiniones online en las estrategias de venta y política de precios. Concluye que las opiniones online pueden incrementar las ventas, reducir la sensibilidad al precio y aumentar la satisfacción postventa informando al cliente sobre los atributos más importantes del producto y reduciendo su incertidumbre a la hora de la compra. Los consumidores que toman una decisión informada están más dispuestos a pagar un precio más alto, están normalmente más satisfechos y se convierten en promotores de la marca online.

Como vemos, son varios los sectores objeto de atención por parte de los investigadores para estudiar el impacto de la reputación online en las empresas. Se puede concluir que la reputación online tiene gran impacto en los resultados económicos de empresas tan variopintas como las compañías aéreas, las páginas web de venta de libros, de videojuegos, de venta de vinos y otras más.

El sector turístico, al igual que otros, ha sufrido una transformación radical en los últimos años, debido principalmente a la introducción de internet a la hora de planificar y reservar el viaje y la influencia del Social Media en los viajeros.

En el campo de los servicios turísticos, debido a la naturaleza de los mismos, la reputación cobra una importancia mayor. Podemos decir que es de vital importancia dado que los productos que se ofrecen en este ámbito son intangibles y no pueden evaluarse antes de su consumo, por lo que la experiencia de otros usuarios resulta ser de gran valor a la hora de tomar una decisión de compra.

Hay varias razones por las que la reputación online es crítica para el sector turístico y hotelero (Litvin, Goldsmith, \& Pan, 2006):

- los productos ofrecidos son intangibles y no pueden ser evaluados antes de su consumo, incrementando por lo tanto la importancia de la influencia interpersonal

- muchos productos hoteleros y turísticos se perciben como compras de alto riesgo, por lo que el riesgo emocional evaluado por el grupo es un aspecto importante del proceso de decisión de compra

- los servicios / productos turísticos y hoteleros sufren de una gran estacionalidad y son perecederos, incrementando por lo tanto los niveles de estrés de marketing para los hoteles 
- el sector es muy competitivo

- dado el estado de la investigación al respecto, el sector está por detrás de otros en el desarrollo y aplicación de estrategias para gestionar la reputación online

Como principal elemento de generación de reputación online de hoteles, deben destacarse las opiniones vertidas por los usuarios en las páginas de reservas de los mismos, o en las dedicadas a la recogida de opiniones y puntuaciones. Incluso pueden ser relevantes las aportadas en otros sitios no especializados en hoteles o viajes (Martínez María-Dolores, Bernal García, \& Mellinas, 2012).

De esta forma, queda patente la importancia de los portales de opinión como auténticas plataformas generadoras de reputación hotelera en la red (Hernández Estárico, Fuentes Medina, \& Morini Marrero, 2012).

Los principales impactos de la reputación online identificados desde la perspectiva del consumidor se encuentran relacionados con aspectos relativos al proceso de decisión de compra, a la aceptación del producto, a la reducción del riesgo en la compra, a la percepción de credibilidad / confianza, a la comparación entre diferentes hoteles, al conocimiento de marca, a la intención de compra y a la lealtad (Salvi, Serra Cantallops, \& Ramón Cardona, 2013).

Los principales impactos de la reputación online desde la perspectiva de las empresas son (Salvi, Serra Cantallops, \& Ramón Cardona, 2013) el control de calidad y puesta en marcha de nuevos procedimientos, posibilidad de ofertar precios más altos, interacción con los consumidores y posibilidad de respuesta para la solución de eventuales problemas, estrategias de marketing específicas para los targets y sus necesidades generando lealtad por parte de los clientes y comparación de la reputación online con la competencia.

Aunque la investigación referente al tema es reciente, ya en varios artículos se abren líneas de investigación sobre los impactos del e-wom ${ }^{4}$ en los resultados de los hoteles. Por ejemplo Vermeulen et al. (2008) afirman que la reputación online de calidad reduce la sensibilidad al precio de los consumidores, lo cual puede llevar a una disminución de la guerra de precios entre hoteles y por lo tanto a márgenes más altos.

En el estudio realizado por comScore y the Kelsey Group (Online Consumer-generated Reviews have significant impact on offline purchase behaviour, 2007) se demuestra que los consumidores están dispuestos a pagar más por un servicio mejor valorado a cambio de un servicio de mejor calidad. Concretamente, los resultados de su estudio indican que los consumidores están dispuestos a pagar como mínimo el $20 \%$ más por servicios que tienen una valoración de «excelente» o una puntuación de 5 sobre 5 , ante el mismo servicio con una valoración de «bueno» o de 4 puntos sobre 5 .

El estudio revela también que el $40 \%$ de los usuarios que vieron las opiniones online de un hotel realizaron una reserva en dicho establecimiento. En concreto, en el caso de los hoteles, el $87 \%$ de los encuestados confirman que las opiniones tuvieron una gran influencia en su decisión de compra.

4 E-wom: Electronic Word of Mouth. Término utilizado en la literatura científica como sinónimo de reputación online, opiniones online, User Generated Content (contenido generado por el usuario), etc. 
Ye et al (2009), demuestra empíricamente que las opiniones online de los usuarios tienen un impacto importante en las reservas hoteleras online. Su estudio se basa en el análisis de los comentarios realizados en la OTA (Online Travel Agency) más importante de China, donde al mismo tiempo se realizan las reservas hoteleras. Sus resultados muestran que las opiniones positivas pueden incrementar significativamente el número de reservas de habitaciones de un hotel: un $10 \%$ de mejora en la puntuación de los usuarios puede incrementar las ventas en un 4,4\% dentro de la OTA.

Yacouel y Fleischer (2010) demuestran que los agentes de viaje cibernéticos, como ellos denominan a las OTAs, que publican valoraciones y opiniones de clientes pueden afectar al precio de mercado. En su investigación prueban que las puntuaciones altas obtenidas mediante opiniones positivas en Booking permiten a los gestores de hotel cargar precios más altos que los competidores con puntuaciones más bajas. Por lo tanto, establecen una relación directa y positiva entre la reputación y el precio.

Este incremento de precio gracias a la buena reputación motiva a los hoteleros a invertir de hecho en mejorar la calidad para aumentar los estándares; es necesario destacar que las OTAs han mejorado la transmisión de la información permitiendo una mejor relación entre precio y calidad.

Tuominen (2011) demuestra en su investigación que hay una relación entre el número de opiniones escritas en Tripadvisor y los resultados del hotel, entendidos como el nivel de ocupación, el precio medio y el RevPAR ${ }^{5}$. Asimismo, demuestra la relación entre la puntuación de las opiniones y los resultados del hotel.

Uno de los aspectos más importantes que aporta este autor es que no encuentra ninguna evidencia para validar una relación entre el índice de popularidad de Tripadvisor y los resultados del hotel. Sin embargo, es necesario destacar la importancia de este índice en términos de visibilidad del establecimiento, ya que los hoteles se presentan por orden de popularidad y por lo tanto se influye en el proceso de decisión de compra.

Chris Anderson (2012) analiza el impacto de los Social Media en los resultados de los hoteles, llegando a las siguientes conclusiones:

1. El porcentaje de consumidores que consultan opiniones en Tripadvisor antes de reservar una habitación de hotel ha ido aumentando con el tiempo, al igual que el número de opiniones que leen dichos viajeros antes de elegir el establecimiento.

2. Si un hotel aumenta su puntuación 1 punto en una escala de 1 a 5 (por ejemplo de 3,3 a 4,3), el hotel puede incrementar su precio un $11,2 \%$ y mantener la misma ocupación o cuota de mercado.

3. El incremento de un $1 \%$ en la puntuación de la reputación online del hotel implica un incremento del 0,89\% en el precio medio del hotel (Average Daily Rate) ${ }^{6}$. Del mismo modo, este aumento del $1 \%$ en la reputación conlleva un incremento de la ocupación de hasta un $0,54 \%$. Finalmente, esta mejora de la reputación en un $1 \%$ implica un incremento en RevPAR de hasta un $1,42 \%$.

5 RevPAR: Revenue Per Available Room. Ingreso por habitación disponible.

6 ADR: Average Daily Rate: precio medio diario de un hotel. Se calcula dividiendo los ingresos de alojamiento entre las habitaciones vendidas. 
Ya en línea con el objeto de nuestro estudio, Ögüt y Onur (2012) estudian el impacto de dos parámetros de calidad (el número de estrellas y la valoración de los clientes) en la venta de habitaciones y en los precios de los hoteles. Con su investigación demuestran que una valoración de clientes más alta incrementa significativamente las ventas online de los hoteles. Así por ejemplo, un 1\% de incremento en la reputación online incrementa los ingresos por habitación hasta un 2,68\% en el caso de París y hasta un 2,62\% en el caso de Londres, las dos ciudades objeto del estudio. Asimismo, demuestran que una mayor categoría (número de estrellas) no implica un incremento en las ventas.

También demuestran empíricamente que los hoteles aplican precios más altos si tienen una valoración más alta y que los precios de los hoteles de mayor categoría son más sensibles a un aumento en la reputación online, incrementándose en mayor medida que en hoteles de categorías inferiores. Así, un aumento de un $1 \%$ en la reputación online incrementa el precio del hotel en un $1,036 \%$ en el caso de París y en un $0,993 \%$ en el caso de Londres.

Otra conclusión muy interesante de su estudio es que los consumidores encuentran las opiniones online de otros usuarios más convincentes que el sistema de estrellas o categorías hoteleras, además de ser un indicador objetivo de la calidad intrínseca de los hoteles. Por lo tanto se podría afirmar que hoy en día las valoraciones online sustituyen en gran parte al sistema de estrellas a la hora de tomar una decisión de compra.

Como afirman los autores, una valoración alta por parte de los clientes a través de las opiniones online puede ser un factor diferenciador para los hoteles, reduciendo la intensa guerra de precios entre hoteles e incrementando por lo tanto sus ventas y sus márgenes.

El precio es una de las variables más importantes de la estrategia de marketing, puesto que una mínima fluctuación en los precios puede tener un impacto importante en la facturación y en la rentabilidad .

Tabla 1

EL IMPACTO DEL PRECIO EN EL RESULTADO DE LAS EMPRESAS

\begin{tabular}{|l|c|c|}
\hline & $\begin{array}{c}\text { Impacto en la rentabilidad } \\
\text { de un 1\% de cambio }\end{array}$ & $\begin{array}{c}\text { \% incremento necesario } \\
\text { para duplicar beneficios }\end{array}$ \\
\hline Incremento en precio & $\mathbf{1 1 , 0 \%}$ & $\mathbf{9 , 1 \%}$ \\
\hline Incremento en ventas unitarias & $3,7 \%$ & $27,1 \%$ \\
\hline Reducción en costes variables & $7,2 \%$ & $13,7 \%$ \\
\hline Reducción en costes fijos & $2,7 \%$ & $37,1 \%$ \\
\hline
\end{tabular}

Fuente: Kohli, C. y Suri, R. The price is right? Guidelines for pricing to enhance profitability, (2011).

Si el impacto del precio tiene esta importancia en la generalidad de las empresas, en el sector hotelero el tema es clave, ya no para la rentabilidad, sino para la supervivencia de la empresa. El entorno enormemente competitivo en el que se mueven los hoteles, la transparencia de precios que ha provocado internet, la rápida caducidad de una habitación de hotel y la obsesión del cliente por encontrar el mejor precio en los canales online hacen que la fijación de precios en el sector hotelero se haya convertido en una mezcla de arte y ciencia altamente compleja. 
En el sector turístico, los métodos aplicados a la fijación del precio se pueden englobar en tres categorías, según Muñoz Oñate (1994):

- los que utilizan criterios económico-empresariales: aquí podríamos destacar la fijación de precios en función de los costes o la fijación de precios en función del margen de rentabilidad esperado

- los que utilizan criterios centrados en el cliente, entre los que destaca el valor del producto percibido

- y los derivados del estudio de la competencia y los mercados, debido principalmente a la alta competencia existente en el sector.

Cada uno de estos métodos, aplicado de forma única, deja de lado información necesaria para tomar la decisión correcta.

En la práctica, no existe un único método para fijar el precio, sino que se utiliza toda la información disponible (referente a costes, mercado y demanda) para tomar la mejor decisión en cuanto al precio. Hoy en día, la fijación del precio en los establecimientos hoteleros es de tal importancia que ha generado una especialidad en sí misma denominada Revenue Management.

El Revenue Management es un conjunto de técnicas de gestión mediante las que se intenta vender el producto adecuado, al precio adecuado, en el momento adecuado, al cliente adecuado y a través del canal de distribución adecuado.

El Revenue Management implica el estudio del producto, de la segmentación, de la competencia y de la distribución, que junto con el análisis de patrones de demanda para realizar previsiones de ventas tiene como fin acertar en el precio ofertado a cada cliente y poder así optimizar los recursos disponibles y maximizar los ingresos.

El Revenue Management implica una política de precios variables, lo que supone ofrecer diferentes precios a clientes por un mismo servicio y al mismo tiempo, basados en las características de la demanda con el objetivo de maximizar la ocupación en períodos de baja demanda y de maximizar el precio medio cuando la demanda es alta (Noone \& McGuire, 2013).

El Revenue Management se aplica a empresas de varios sectores, no sólo del hotelero, pero todas ellas tienen determinadas características comunes (Kimes, 1989):

- tienen capacidad fija

- su inventario es perecedero

- sus costes fijos son muy altos comparados con el coste marginal de venta de una unidad

- su demanda es estacional

- la reserva del servicio se realiza normalmente antes de su disfrute

- su segmentación es heterogénea

Dichas características se encuentran en un gran número de empresas del sector turístico: hoteles, líneas aéreas, empresas de alquiler de coches, salas para eventos y congresos, compañías de cruceros, campos de golf, etc. Pero también en empresas de 
otros sectores: publicidad, telefonía, salas de cine, etc. Es por ello que la importancia del Revenue Management está creciendo en los últimos años por la necesidad de optimizar los recursos de la empresa.

De otro lado, internet ha cambiado la forma de establecer las políticas de precios por parte de los hoteles (Phillips, 2005). En primer lugar internet incrementa la velocidad de las decisiones de precios: hoteles que antes cambiaban los precios una vez al trimestre ahora los tienen que cambiar una vez al día, con el fin de adecuarse a los vaivenes de la demanda y de la competencia. En segundo lugar internet pone a disposición de la empresa de manera inmediata una gran cantidad de información sobre el comportamiento del consumidor: qué ha comprado, cuándo, cuánto, durante cuánto tiempo ha estado en la web, etc. En tercer lugar, internet es un laboratorio de experimentación para probar diferentes alternativas y estrategias de precios en tiempo real, puesto que las respuestas de los clientes se pueden evaluar de manera inmediata. Y en cuarto y último lugar, Internet proporciona información sobre costes y precios de la competencia.

Este cambio que ha supuesto Internet en la forma de decidir los precios se ve reforzado por la creciente importancia de las opiniones online.

A pesar de la relevancia del tema, se han realizado pocos estudios que se centren específicamente en la influencia de la reputación online en la política de precios de la empresa.

Kohli y Suri (2011) afirman que en la medida en que es fácil comparar precios y calidad, los precios más altos sólo se pueden fijar de manera proporcional al valor añadido. Y curiosamente, este valor añadido se puede basar en atributos, ventajas o imagen corporativa. Es aquí donde podemos destacar la relación entre la reputación corporativa y la estrategia de precios: una buena reputación supone un valor añadido para el cliente y por lo tanto dicho cliente está dispuesto a pagar más.

Ya más en la línea de nuestra investigación, Helm (2013) estudia la influencia de la reputación corporativa en las reacciones de los clientes a los incrementos de precios en el sector de las líneas aéreas. Concluye Helm en su estudio que las empresas deberían tener en cuenta la reputación corporativa en sus estrategias de precios, puesto que un análisis de la reputación puede ayudar a la hora de prever reacciones del consumidor a incrementos de precio, al tiempo que aumentar la rentabilidad. Entre sus resultados más importantes destacan:

- Cuanto más favorable es la reputación percibida, menos probable es que los clientes perciban negativamente un incremento de precio.

- Cuanto más favorable sea la percepción del cliente sobre la reputación corporativa, más alta será la percepción referente a la equidad del precio.

- Cuanto más alta sea la percepción de la reputación corporativa, más alta será la intención de compra del cliente

En definitiva, es rentable para las empresas invertir en su reputación, puesto que una buena reputación amortigua las repercusiones negativas de los incrementos de precios. Este hecho es especialmente importante en los sectores que utilizan políticas de precios dinámicos y con una competencia dispuesta a entrar en guerra de precios (como las líneas aéreas o los hoteles). 
Ya en el campo que nos ocupa, Noone et al (2011) estudia las oportunidades que surgen gracias al los Social Media para el Revenue Management, y por lo tanto, la influencia que tiene el Social Media en la política de precios de los hoteles, entre otros factores.

En su estudio, Noone clasifica los impactos del Social Media en función de dos variables que dan lugar a cuatro cuadrantes. Por una parte el flujo de información, que se divide en el proveniente del cliente (opiniones online, valoraciones, fotos, comentarios...) y en el que proviene de la empresa (promociones, ofertas y notas de prensa emitidas a través de canales de Social Media, o respuestas a los comentarios de los clientes). Por otra parte está el plazo en el que tiene efecto dicha información y que se divide en corto plazo (decisiones operativas) y largo plazo (decisiones estratégicas).

Tabla 2

IMPACTO DEL SOCIAL MEDIA EN EL REVENUE MANAGEMENT

\begin{tabular}{|l|l|c|c|}
\cline { 3 - 4 } \multicolumn{1}{|c|}{} & \multicolumn{2}{c|}{ Plazo de ejecución } \\
\cline { 2 - 4 } & A corto plazo & A largo plazo \\
\hline $\begin{array}{l}\text { Fuente de } \\
\text { información }\end{array}$ & $\begin{array}{c}\text { Proveniente } \\
\text { del cliente }\end{array}$ & $\begin{array}{c}\text { Proporcionar información } \\
\text { para decisiones promocionales } \\
\text { y de precios }\end{array}$ & $\begin{array}{c}\text { Proporcionar información para } \\
\text { el desarrollo de estrategias } \\
\text { de políticas de precios, CRM } \\
\text { (Customer Relationship } \\
\text { Management) y gestión de los } \\
\text { canales de distribución }\end{array}$ \\
\cline { 2 - 4 } & $\begin{array}{c}\text { Proveniente } \\
\text { de la } \\
\text { empresa }\end{array}$ & $\begin{array}{c}\text { Gestionar la demanda e } \\
\text { incrementar la notoriedad de } \\
\text { marca }\end{array}$ & $\begin{array}{c}\text { Gestionar la retención y el } \\
\text { incremento de clientes }\end{array}$ \\
\hline
\end{tabular}

Fuente: Noone, McGuire, \& Rohlfs, Social media meets hotel revenue management: Opportunities, issues and unanswered questions, 2011.

Pues bien, el contenido generado por el usuario tiene efectos en la política de precios tanto a corto como a largo plazo. En el caso del corto plazo, el UGC (User Generated Content), entre el que se encuentran las opiniones online, influye a la hora de tomar decisiones tácticas de precios. Dichas opiniones proporcionan al hotel información de lo que quieren sus clientes y de cuánto están dispuestos a pagar, lo que permite al hotelero reaccionar de una forma absolutamente dinámica a las necesidades del mercado en cuanto al precio.

El UGC también influye en las decisiones estratégicas de precio a largo plazo. Las decisiones de precios se toman teniendo en cuenta una serie de variables como el nivel de demanda, la sensibilidad al precio de dicha demanda y el posicionamiento con respecto a la competencia. Es en esta última variable en donde el UGC influye de manera más notoria, puesto que el carácter público de dicha información junto con la transparencia de precios provocada por Internet hace que cada hotel tenga toda la información necesaria para diferenciarse de su competencia en base al precio y a sus atributos. 


\section{Figura 1 \\ INFLUENCIA DEL SOCIAL MEDIA EN LA POLÍTICA DE PRECIOS}

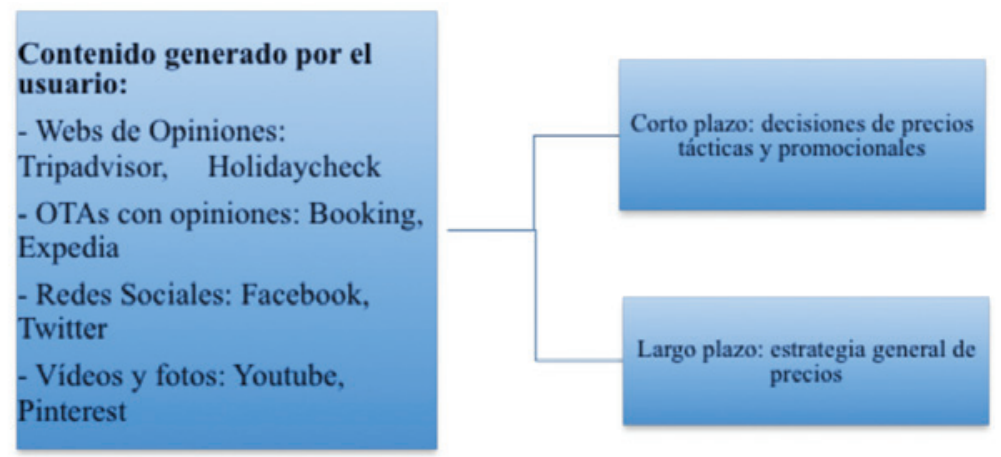

Fuente: Noone, McGuire, \& Rohlfs, Social media meets hotel revenue management: Opportunities, issues and unanswered questions, 2011

En un estudio posterior, Noone et al. (2013) estudia la influencia que tienen la política de precios variables derivada del Revenue Management y el UGC (User Generated Content) en la decisión de compra del cliente. Su investigación concluye con dos resultados principales. Por una parte, si hay UGC, el precio no tiene un impacto significativo en la calidad percibida en el momento de la compra. En cambio, la información derivada de las opiniones y de las puntuaciones juega un papel significativo en la percepción de calidad de los clientes.

Por otra parte, el precio y el UGC tienen efectos significativos en el valor percibido, aunque los consumidores confían más en las opiniones que en las puntuaciones a la hora de evaluar la relación precio-beneficio.

Por ello, una de las más claras consecuencias que tienen las opiniones online en la política de precios de un establecimiento hotelero es que el precio no se puede ser la única base sobre la que competir en el mundo actual.

Estamos viendo a lo largo de todo el estudio que la decisión de compra de un cliente no se basa únicamente en el precio, sino también en otros atributos. Noone (2013) demuestra que en el ámbito de los hoteles los consumidores integran la información de precio con otra información que no tiene nada que ver con el precio para hacerse una idea del valor total de la compra. Además estos consumidores están dispuestos a pagar un precio más alto si la información que no tiene que ver con el precio sugiere una experiencia superior. Esta información que no tiene que ver con el precio surge de tres fuentes: el UGC, es decir, las opiniones online y las valoraciones cuantitativas; los rankings derivados del UGC (por ejemplo, el índice de popularidad de Tripadvisor) y la marca.

A la hora de establecer la política de precios de un hotel es necesario entender cómo los clientes integran toda esa información con el precio a la hora de decidir su compra. Dada la inmediata disponibilidad de este tipo de información a la hora de la compra, cualquier estrategia de precios que no tenga en cuenta el valor percibido del producto por parte del cliente no tendrá la eficacia deseada. 
Como conclusión muy interesante y desde otro punto de vista a nuestro estudio, un precio más bajo puede no ser suficiente para contrarrestar el impacto de las opiniones negativas, lo que implica claramente la gran relación que hay entre las opiniones y la política de precios.

Según Noone et al., (2013) los hoteles deben prestar atención a más factores que al solo precio en relación con sus competidores. Una estrategia de precios efectiva debe incluir también el posicionamiento en cuanto a opiniones dentro del competitive set, aunque ello no significa necesariamente que un buen posicionamiento debe conllevar una subida de precios, puesto que en esta decisión influyen otros muchos factores: patrones de demanda, propuesta de valor, posición competitiva, etc.

Avanzando un poco más en el tema, Blal y Sturman (2014) analizan el diferente efecto que tiene el e-WOM ${ }^{7}$ según el segmento en que se encuadre el hotel, desglosando además dos dimensiones en el e-WOM: la valencia, entendida como la puntuación obtenida mediante las opiniones y el volumen, entendido como el número de opiniones sobre un hotel. Sus resultados se pueden aplicar indistintamente tanto a hoteles urbanos y vacacionales como a hoteles independientes o pertenecientes a cadenas, y se pueden resumir en lo siguiente:

- en el caso de los hoteles de lujo y los hoteles de gama alta, la valencia (la puntuación de las opiniones) tiene una influencia muy alta en las ventas de los hoteles, mientras que el volumen de opiniones es indiferente o incluso perjudicial si es muy alto.

- en el caso de los hoteles de gama media y baja, sin embargo, un incremento en el número de opiniones tiene un efecto directo en los resultados de ventas del hotel.

Estos resultados indican que el e-WOM no tiene la misma influencia en las ventas en todos los hoteles, sino que depende del segmento donde se encuadre el hotel.

En definitiva, vemos que la investigación en este campo es muy reciente y que en general los principales resultados indican una relación directa entre la reputación online de un hotel y su política de precios.

Tras el análisis del estado del arte y como consecuencia de las aportaciones previas realizadas sobre el tema, nos planteamos dos hipótesis que serán contrastadas a lo largo del estudio.

H1: Los hoteles situados en primera posición en el ranking de Tripadvisor tienen precios de venta más altos que los competidores de su misma zona geográfica y misma categoría

H2: Los hoteles con buena reputación online la tienen en cuenta como variable a la hora de establecer su política de precios.

\section{METODOLOGÍA}

Una vez revisada la literatura sobre el tema en cuestión, es preciso analizar empíricamente la información disponible para poder contrastar las hipótesis presentadas en el apartado anterior.

7 E-Wom: Electronic Word of Mouth - boca a oreja electrónico. 
Hasta el momento hemos visto la enorme importancia que tiene la reputación online en la empresa a todos los niveles. Ahora bien, ¿qué referencia podemos tomar dentro del sector turístico para conocer la reputación online de un establecimiento hotelero?

Hoy en día, los viajeros pueden compartir sus opiniones y valoraciones en infinidad de Social Media específicos de este sector. La mayoría de las OTAs (Booking, Expedia...) ofrecen ya el apartado de valoraciones de otros usuarios; existen también páginas web exclusivamente para comentarios de usuarios (Tripadvisor, Holidaycheck); las redes sociales como Facebook y Twitter permiten acceder rápidamente a las opiniones o comentarios de usuarios y las redes sociales de contenido audiovisual (Youtube, Pinterest) permiten a los consumidores acceder a vídeos y fotos reales hechos por otros usuarios durante su estancia.

Dentro de este maremágnum de comentarios y valoraciones que podemos encontrar con respecto a un hotel en la red, destaca por su volumen Tripadvisor.

Según Market Metrix, empresa líder en la monitorización de comentarios online del sector hotelero, Tripadvisor es la página de comentarios líder en el mundo. Cierto es que hay diferencias regionales, destacando la región Asia Pacífico como aquella en que Tripadvisor tiene menor importancia que otras fuentes a la hora de valorar comentarios sobre un hotel.

Figura 2

\section{PRINCIPALES PÁGINAS DE COMENTARIOS ONLINE EN EL SECTOR HOTELERO}

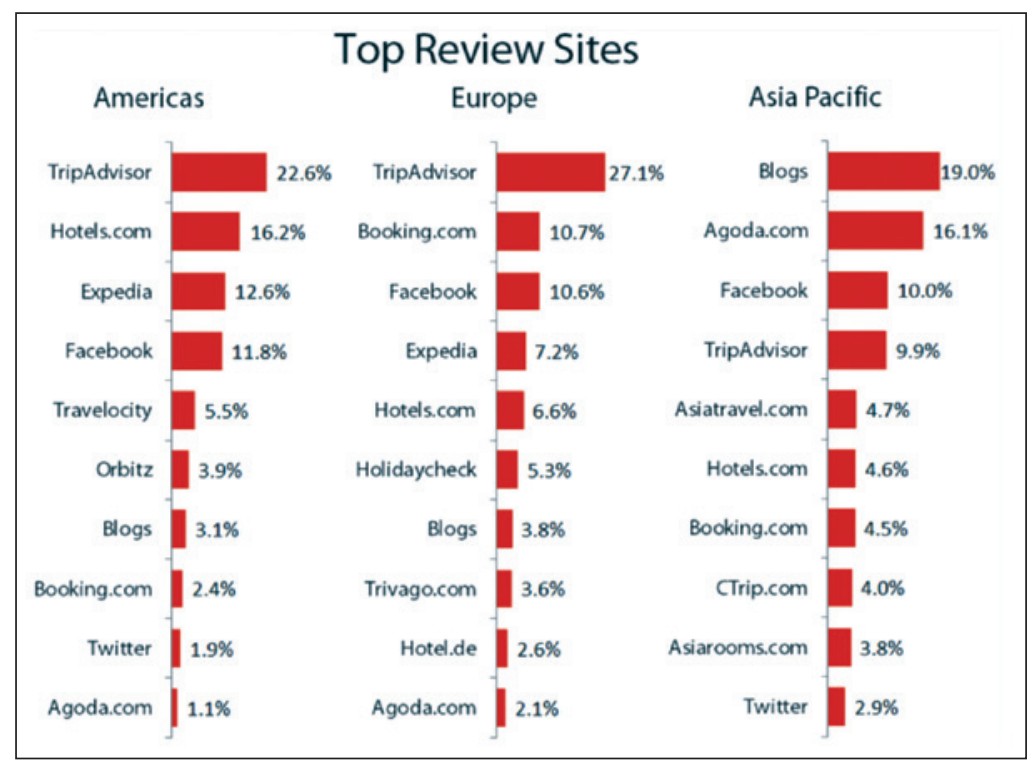

Fuente: Market Metrix (Review Sites: it's a regional thing, 2013).

Como se puede observar en la figura 2, en la región de las Américas y en Europa, Tripadvisor se sitúa en el primer lugar dentro de las páginas web/Social Media que recogen contenidos de los usuarios, seguida en ambos casos por la OTA dominante en la zona: 
Hotels.com y Booking.com. Es interesante observar que Facebook, la red social más importante, ocupa el cuarto lugar en el caso de las Américas y el tercero en el caso de Europa.

Es indudable que Tripadvisor representa la principal fuente de reputación online del sector turístico y más en concreto, del sector hotelero.

En su informe global de abril 2014 (TripBarometer 2014 Global Report), Tripadvisor estima que tres cuartas partes de los viajeros utiliza Tripadvisor como fuente de inspiración para sus vacaciones; es la fuente más influyente seguida de cerca por el boca-oreja. Por otra parte, destaca que el precio es el factor más importante a la hora de elegir establecimiento hotelero, aunque los hoteles perciben que las opiniones online tienen un impacto mayor que el precio.

Así, la práctica totalidad de los viajeros $(98 \%)$ buscan en internet ideas e inspiración para sus vacaciones y la mayoría lo hace en Tripadvisor (93\%).

Entre los factores que tienen influencia en la decisión de reservar alojamiento por parte de los consumidores, destacan el precio en primer lugar y las opiniones online de otros viajes que hayan estado en el establecimiento en segundo lugar. Es necesario recalcar que cuando se realizó el mismo estudio el año anterior, la ubicación era el segundo factor más influyente a la hora de reservar un alojamiento. Ello nos confirma la creciente importancia de las opiniones online en el proceso de decisión de compra.

\section{Figura 3 \\ FACTORES QUE INFLUYEN EN LA DECISIÓN DE RESERVAR ALOJAMIENTO}

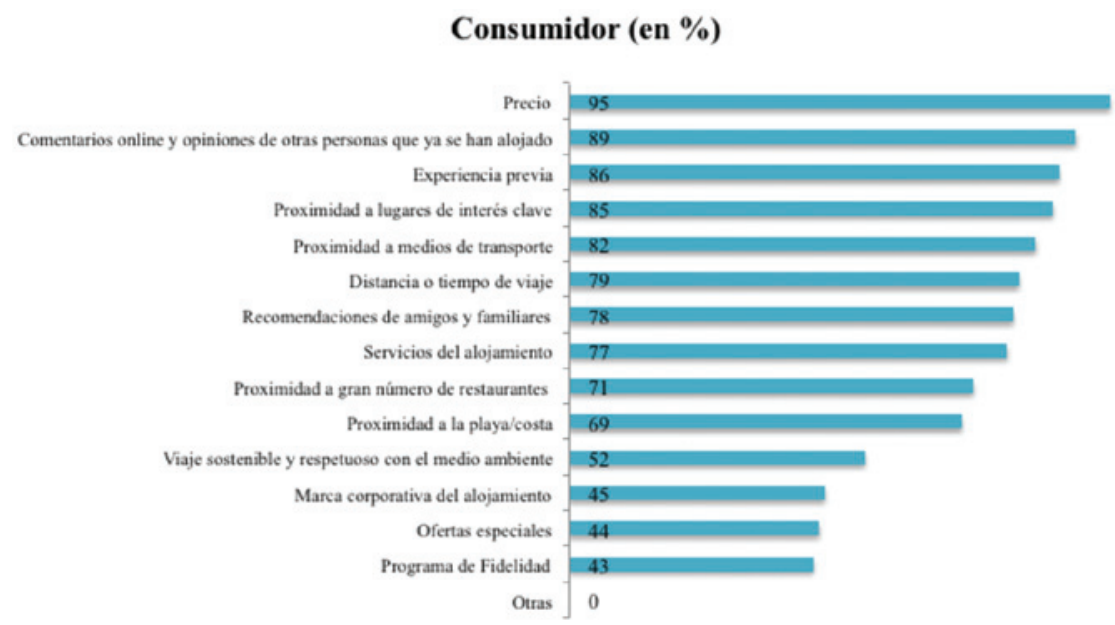

Fuente: Tripbarometer 2014.

En definitiva, como hemos podido constatar, Tripadvisor lidera el mercado de opiniones sobre alojamientos hoteleros y los gestores de establecimientos lo tienen en cuenta a la hora de establecer sus acciones de marketing externo, fidelización de clientes, marketing interno, gestión de reputación y políticas de precios. 
Una vez demostrada la importancia que tiene Tripadvisor como referente mundial de la reputación online de los hoteles, vamos a centrarnos en el Índice de Popularidad de Tripadvisor, parámetro que utilizaremos en el apartado empírico de este trabajo como medida de reputación online.

El índice de popularidad de Tripadvisor representa la fuente dominante en cuanto a ranking de hoteles derivado del contenido generado por el usuario. (Noone \& McGuire, 2013)

El índice de popularidad de Tripadvisor está basado en las opiniones de los usuarios y refleja el posicionamiento de un hotel con respecto a los hoteles similares situados en la misma zona geográfica.

El índice de popularidad es importante, puesto que cuanto más cercano esté un hotel de la primera posición, mayor será la visibilidad del hotel ante potenciales clientes que buscan alojamiento en la zona. Además, la cantidad de opiniones positivas de otros viajeros influirá en la decisión de compra.

Los principales factores que conforman el índice de popularidad son los siguientes:

- la calidad de las opiniones: cuanto más positivas sean, más alto el ranking

- la cantidad de las opiniones: cuantas más opiniones, más alto el ranking

- la antigüedad de las opiniones: las opiniones más recientes tienen mayor peso a la hora de posicionar mejor el hotel.

Esta posición es dinámica y varía a lo largo del tiempo, puesto que depende también de los comentarios recibidos por los demás hoteles de la misma zona. Además, un mismo establecimiento puede tener diferentes posiciones según el ámbito geográfico de búsqueda.

Según el mismo Tripadvisor, la clasificación por estrellas de los hoteles y las relaciones comerciales que los hoteles mantengan con Tripadvisor no influyen en el índice de popularidad.

Por todo lo anteriormente expuesto es por lo que hemos elegido el índice de popularidad de Tripadvisor como medida de la reputación online de un establecimiento hotelero.

En cuanto al trabajo de investigación, se han seleccionado cuatro ciudades españolas con un volumen importante de negocio hotelero urbano: Madrid, Valencia, Sevilla y Bilbao. Dentro de cada una de las ciudades se ha elegido al hotel situado en la primera posición según el Ranking de Tripadvisor. Es importante destacar que se han elegido destinos urbanos y no vacacionales porque la homogeneidad de los hoteles es mayor en un destino urbano. En un hotel vacacional, por el contrario, hay mucha diferenciación en cuanto al producto en sí mismo, lo cual nos hubiera llevado a resultados difíciles de elaborar.

El hotel elegido no ha sido el primero en términos absolutos, sino el posicionado primero dentro de la categoría de cuatro estrellas en dicho destino. Se ha seleccionado dicha categoría porque en todos los destinos era la que englobaba un mayor número de hoteles similares. Es decir, a los efectos de este estudio, entendemos como hotel situado en primera posición de un destino aquel hotel de cuatro estrellas con un número mínimo de 25 habitaciones situado en el primer puesto por encima de los demás hoteles de las mismas características. 
A continuación se ha elegido el set de competidores de cada uno de los cuatro hoteles de referencia basándonos en la selección natural de una de las OTAs ${ }^{8}$ de mayor volumen en el mercado internacional: Booking.com. Al seleccionar un hotel concreto en Booking para unas fechas determinadas, el sistema ofrece la descripción del alojamiento, fotos, tipos de habitaciones y tarifas. Además, en la parte inferior de la página, Booking inserta una frase: «Los clientes que han consultado el Hotel X también han consultado...» y ofrece el nombre de varios hoteles de igual o similar categoría y servicios, con sus descripciones respectivas. Entendemos por lo tanto que la selección de Booking corresponde al competitive set ${ }^{9}$ real de los hoteles de referencia, puesto que se trata de hoteles que los clientes consultan en el momento de la compra. Se han seleccionado por lo tanto, para cada hotel de referencia, tres hoteles competidores basados en los resultados ofrecidos por Booking, siendo hoteles de la misma categoría, misma o similar ubicación y en definitiva un tipo similar de producto.

Se eligen por lo tanto 16 establecimientos hoteleros en cuatro destinos urbanos diferentes y se monitoriza el precio de venta de cada uno de ellos durante un período de 30 días para un rango de fechas de cuatro meses.

Para ello, dada la imposibilidad de realizar la monitorización de manera manual, hemos contado con la colaboración de BITREVENUE, una empresa tecnológica del sector hotelero que proporciona a los hoteles de manera continua y automática información de los precios de su propio hotel y de sus hoteles competidores en la página de Booking para un período de cuatro meses.

La selección de hoteles y sus competidores se ha realizado en la última semana de marzo de 2014, pero hay que tener en cuenta que el posicionamiento basado en el Ranking de Tripdavisor va cambiando en función de parámetros establecidos por el mismo Tripadvisor.

Los hoteles monitorizados y su correspondiente set competitivo se recogen en la tabla 3:

\section{Tabla 3}

\section{HOTELES SELECCIONADOS POR DESTINO}

\begin{tabular}{|c|c|c|c|c|}
\hline & MADRID & VALENCIA & SEVILLA & BILBAO \\
\hline HOTEL No 1 & Hotel Atlántico & $\begin{array}{c}\text { Hotel Meliá } \\
\text { Valencia }\end{array}$ & Hotel Casa 1800 & Hotel Miró \\
\hline COMP A & Hotel Preciados & Hotel Dimar & $\begin{array}{c}\text { Hotel Vincci La } \\
\text { Rábida }\end{array}$ & $\begin{array}{c}\text { Hotel Hesperia } \\
\text { Bilbao }\end{array}$ \\
\hline COMP B & Hotel Emperador & $\begin{array}{c}\text { Hotel Sercotel } \\
\text { Sorolla Palace }\end{array}$ & $\begin{array}{c}\text { Hotel Fontecruz } \\
\text { Sevilla }\end{array}$ & Hotel Ercilla \\
\hline COMP C & $\begin{array}{c}\text { Hotel Catalonia } \\
\text { Gran Vía }\end{array}$ & $\begin{array}{c}\text { Hotel Ayre Astoria } \\
\text { Palace }\end{array}$ & $\begin{array}{c}\text { Hotel Casas de la } \\
\text { Judería }\end{array}$ & $\begin{array}{c}\text { Hotel NH Villa de } \\
\text { Bilbao }\end{array}$ \\
\hline
\end{tabular}

Fuente: elaboración propia.

8 OTA: Online Travel Agency - Agencia de Viajes Virtual: Booking, Expedia, HRS...

9 Competitive set: conjunto de hoteles competidores 
En cuanto al rango temporal, se han monitorizado los precios de los 16 hoteles diariamente desde el 1 al 30 de abril de 2014, para cada uno de los días comprendidos entre el 1 de abril y el 31 de julio 2014. En total el número de precios monitorizados ha superado los 58.000 items.

Para monitorizar los precios, se han seguido unos criterios homogéneos y comparables en todos los hoteles, debido a variedad de tipos de habitación y tipos de tarifa disponibles en la web. Así, se ha elegido el tipo de habitación doble, común a todos los establecimientos, con ocupación de dos personas, para una noche de estancia. En cuanto al tipo de tarifa, el sistema lo que ofrece es el mejor precio disponible (léase el más económico), sin discriminar entre las condiciones de tarifa, que pueden ser diferentes según los casos. Hay tarifas que exigen prepago total a la hora de la reserva, tarifas que exigen el pago de una noche y tarifas que se pueden cancelar hasta el mismo día. Entendemos que a los efectos de nuestro trabajo es necesario comparar precios con condiciones homogéneas, por lo tanto se ha elegido la tarifa denominada BAR (Best Available Rate $)^{10}$, común a todos los establecimientos y en la que las condiciones de prepago y cancelación son las mismas en todos los hoteles. Para ello ha sido necesario elaborar manualmente los datos ofrecidos por el sistema para poder comparar los precios en igualdad de condiciones.

La tabla 3 es un ejemplo de hoja de cálculo con la toma de precios, disponibilidad de habitaciones y condiciones de tarifa con la que se ha elaborado la parte empírica de este trabajo.

Para homogeneizar los precios y comparar tipos de habitación y tarifas con iguales condiciones, se ha establecido un filtro en el informe original en la columna «tipo de habitación», para eliminar las habitaciones no comparables (por ejemplo suites, junior suites, triples, etc.).

Una vez «depurado» el tipo de habitación, se ha establecido otro filtro en la columna «tipo de tarifa», para separar las tarifas BAR (es decir, cancelación gratuita, no necesario prepago) de las tarifas no reembolsables. El sistema de Bitrevenue toma por defecto el precio más económico, con lo que en unos casos este precio es una tarifa BAR (sin restricciones) y en otros casos se trata de tarifas no reembolsables (con descuento pero con restricciones). Normalmente los hoteles ponen a la venta los dos tipos de tarifa simultáneamente, con el fin de llegar a más segmentos de mercado.

Las tarifas no reembolsables se calculan normalmente con un porcentaje de descuento sobre la tarifa BAR del momento. Así, se ha monitorizado el \% de descuento de cada uno de los hoteles a través del mismo Booking (fuente de datos) o bien de sus propias páginas web. Este descuento oscila entre un $10 \%$ y un $15 \%$ en los hoteles, con lo que en cada hotel se ha realizado el cálculo correspondiente para igualar todas las tarifas y poder compararlas en igualdad de condiciones.

Con los datos ofrecidos por BITREVENUE, por lo tanto, se han elaborado diversas hojas de cálculo para filtrar los datos y poder presentar los resultados de este estudio.

10 BAR: Best Available Rate. Es la mejor tarifa disponible para cualquier cliente en el momento de la compra. 


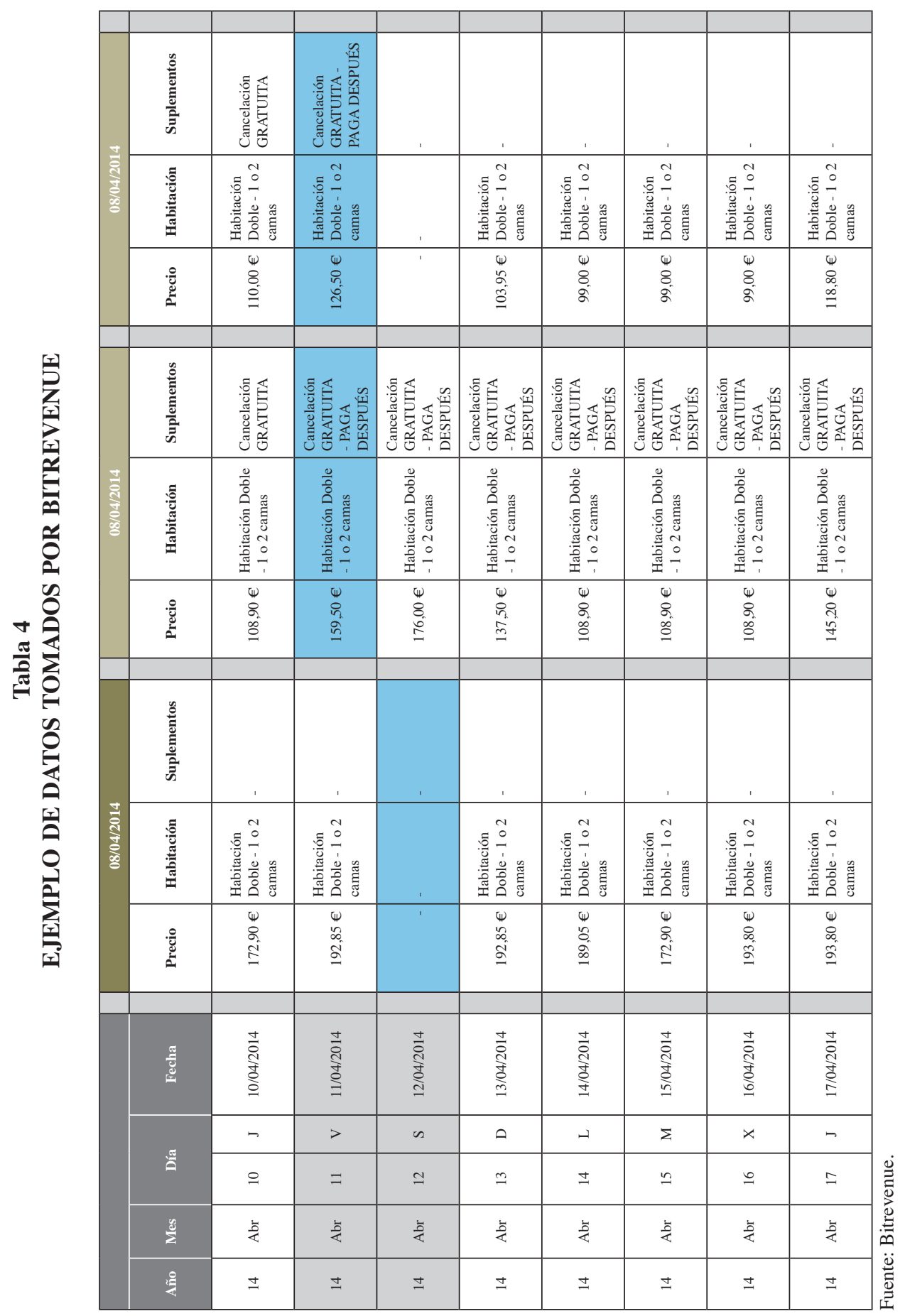




\section{RESULTADOS}

Tras la recopilación y el estudio de la documentación científica que hemos encontrado referente al tema en concreto y mediante el análisis de los datos empíricos seleccionados, podemos concluir con un primer resultado clarísimo: el hotel situado en primera posición en el Ranking de Tripadvisor de un destino concreto vende de media un 22,26\% más caro que su set de competidores en el mismo destino. Analizando durante 4 meses el precio medio de venta de una habitación doble en tarifa BAR para una noche de estancia, y a pesar de las diferencias entre destinos, en todos los casos el hotel posicionado en primer lugar tiene unos precios medios de venta superiores a los de su competitive set.

Las tablas 1,2,3 y 4 como esta, como siempre: minúscula y mayúscula

Tabla 5

PRINCIPALES RESULTADOS: PRECIO MEDIO Y DIFERENCIAS

\begin{tabular}{|l|r|r|r|r|r|}
\cline { 2 - 6 } \multicolumn{1}{c|}{} & MADRID & VALENCIA & SEVILLA & BILBAO & \multicolumn{1}{c|}{ MEDIA } \\
\hline HOTEL 1 & $181,29 €$ & $87,35 €$ & $182,46 €$ & $137,99 €$ & $147,27 €$ \\
\hline COMPSET & $125,10 €$ & $86,84 €$ & $168,81 €$ & $101,07 €$ & $120,46 €$ \\
\hline DIFERENCIA MEDIA & $56,20 €$ & $0,51 €$ & $13,64 €$ & $36,92 €$ & $26,82 €$ \\
\hline DIF MEDIA EN \% & $44,92 \%$ & $0,59 \%$ & $8,08 \%$ & $36,53 \%$ & $22,26 \%$ \\
\hline DIFERENCIA MÁXIMA & $93,27 €$ & $59,01 €$ & $114,84 €$ & $83,93 €$ & $93,64 €$ \\
\hline
\end{tabular}

Fuente: elaboración propia con datos de Bitrevenue

Como vemos en la figura 4, el precio medio del hotel situado en primera posición en Tripadvisor (línea azul) es, excepto en contadas ocasiones, superior al precio medio de su set de competidores.

Las tablas 1, 2 y 3 como esta, como siempre: minúscula y mayúscula

\section{Figura 4 \\ CURVA DE PRECIO MEDIO DE VENTA DEL HOTEL SITUADO EN $1^{a}$ POSICIÓN Y SU COMPSET}

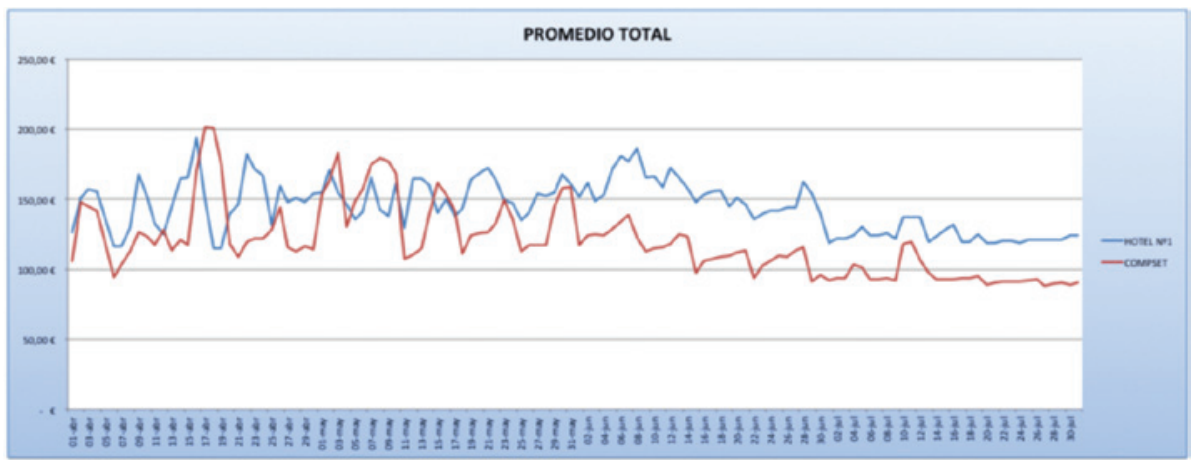

Fuente: elaboración propia con datos de Bitrevenue. 
Es interesante observar en la curva del precio medio ofertado para una habitación doble, que es a más largo plazo cuando la diferencia entre el precio del hotel situado en primera posición y el compset se hace más evidente. Lógicamente, a corto plazo priman las decisiones tácticas y hay momentos en los que, a pesar de ser el hotel con mejor reputación de la ciudad, es necesario bajar el precio para captar demanda.

La diferencia de precio entre el precio ofertado por el hotel situado en $1^{\text {a }}$ posición y el competitive set es de 26,82 euros de media, siendo la diferencia abismal en Madrid (56,20 euros) y mínima en Valencia (0,51 euros).

Dentro de estas medias, hay variaciones en función del destino. Así por ejemplo, en Madrid se genera una diferencia de hasta 93,27 euros entre el precio de una habitación doble en el hotel situado en primera posición y la media de precios del competitive set. En Valencia, la variación máxima se sitúa en 59,01 euros, en Sevilla la máxima está en 114,84 euros y en Bilbao se encuentra en 83,93 euros.

Analizados los datos recopilados, podemos afirmar que se cumple las primera de las dos hipótesis propuestas en este trabajo: H1: Los hoteles situados en primera posición en el ranking de Tripadvisor tienen precios de venta más altos que los competidores de su misma zona geográfica y misma categoría.

En cuanto a la segunda hipótesis (H2: Los hoteles con buena reputación online la tienen en cuenta como variable a la hora de establecer su política de precios), es necesario recalcar que los datos no ofrecen resultados concluyentes al respecto, si bien se puede inferir que los precios más altos que imponen los hoteles situados en primer lugar en el ranking de Tripadvisor se deben en parte a la buena reputación online.

La diferencia de nuestro estudio con los precedentes es fundamentalmente la orientación. En la revisión de la literatura anterior específica sobre el tema, se analiza principalmente el impacto de la reputación online sobre las ventas o sobre los datos finales del establecimiento (ADR, Ocupación, RevPAR). Nosotros sin embargo, hemos querido abrir una nueva línea para investigar si los hoteles, desde el punto de vista del establecimiento de sus políticas de precios, tienen en cuenta la reputación online.

\section{CONCLUSIONES, LIMITACIONES Y FUTURAS LÍNEAS DE INVESTIGACIÓN}

A lo largo de este trabajo se ha intentado demostrar que la reputación online es una variable fundamental a la hora de establecer las políticas de precios de los establecimientos hoteleros. Si bien los datos examinados durante la investigación ofrecen resultados concluyentes (todos los hoteles situados en primer lugar tienen precios de venta más altos que su set de competidores), no podemos afirmar basados únicamente en los resultados que dichos precios de venta más altos se deben exclusivamente a una buena reputación online.

Sin embargo, como consecuencia de la revisión de la literatura, sí se puede afirmar que la reputación online es una variable de gran relevancia a la hora de establecer las políticas y estrategias de precios de cualquier empresa, no sólo de la hotelera.

La gran limitación que nos encontramos a la hora de afrontar un estudio de estas características es la falta de concienciación por parte del sector de las variables que influyen en su política de precios. 
En cualquier caso, tras la lectura y el análisis de los datos del presente trabajo surgen diversas reflexiones que se pueden resumir en las siguientes:

- La reputación online es un factor que hay que tener en cuenta a la hora de establecer las políticas de precios de un establecimiento hotelero, tanto tácticas como estratégicas.

- Estar situado en los primeros puestos dentro de un destino permite al establecimiento ofrecer al cliente precios más altos que los de su competencia

- Una buena reputación online provocará, si se gestiona bien, un incremento en el precio medio del hotel y por lo tanto, si no decrece la ocupación, un incremento en el ingreso por habitación disponible

- Hay hoteles que ya tienen en cuenta su posicionamiento en cuanto a reputación online se refiere como una variable más a la hora de decidir sus precios. Al mismo tiempo, hay una gran mayoría de hoteles para los que esta variable está todavía lejos de ser aplicable en sus estrategias de precios.

- Es necesario una concienciación por parte de todo el sector de que la inversión en calidad y en fidelización del cliente a través de los comentarios online tiene sus repercusiones positivas en los beneficios.

- Los hoteles necesitan invertir en mejorar (o mantener) su reputación online puesto que está demostrada la importancia de esta variable como factor de decisión de compra del cliente.

En definitiva, el hecho de demostrar que en el sector hotelero una buena reputación incrementa los beneficios de las empresas es muy clarificador y cada vez es más necesario que los hoteles lo tengan en cuenta a la hora de establecer sus estrategias de precios.

Vemos sin embargo, que hay muchas carencias de investigación en el tema, así como falta de concienciación por parte del sector en la fundamental importancia de la reputación online en toda la gestión de un establecimiento hotelero.

Así, se abren muchas cuestiones interesantes que pueden constituir futuras líneas de investigación. En primer lugar, sería necesario estudiar en qué medida el hotel utiliza la reputación online a la hora de establecer su política de precios, es decir, si se puede aplicar un «factor de reputación» al precio establecido originalmente, de forma que se incremente o disminuya ese precio.

Por otra parte, sería muy interesante comprobar si el cliente tiene la misma sensibilidad al precio ante un hotel situado en la posición número 1 de la zona geográfica vs la sensibilidad que tendría ante el precio de otros hoteles similares con peor reputación.

Otra línea de investigación se puede abrir para comprobar si los hoteles situados en primera posición en Tripadvisor obtienen una mayor rentabilidad que su competitive set, o si ese incremento de precio les resta cuota de mercado en mayor medida y por lo tanto su rentabilidad es menor.

También sería interesante comprobar si los resultados obtenidos en este estudio se pueden aplicar a los hoteles situados ya no sólo en primera posición, sino en la primera página de Tripadvisor. La primera página contiene por defecto los 30 primeros hoteles 
de cada zona geográfica según el índice de popularidad: ¿afecta igual a la percepción de reputación una posición 3 que una posición 5 ? ¿El estar ya en la primera página presupone una buena reputación que permita un incremento de precio?

Estas y otras cuestiones se irán resolviendo poco a poco, puesto que este tema acaba prácticamente de empezar y tiene muchísimas consecuencias en la correcta gestión de un establecimiento y por lo tanto en su rentabilidad.

\section{BIBLIOGRAFÍA}

ÖGÜT, H., \& ONUR TAS, B. (2012): «The Influence of internet customer reviews on the online sales and prices en hotel industry». The Service Industries Journal , 32 (2), 197-214.

ANDERSON, C. (2012): «The Impact of Social media on Lodging Performance». Cornell Hospitality Report , 12 (15).

BLAL, I., \& STURMAN, M. (2014): «The Differential Effectos of the Quality and Quantity of Online Reviews on Hotel Room Sales». Cornell Hospitality Quarterly , 1-11.

CHEVALIER, J., \& MAYZLIN, D. (2006): «The effect of word of mouth on sales: online book reviews». Journal of Marketing Research , 43 (3), 345-354.

CHUN, R. (2005): «Corporate reputation: meaning and measurement». International Journal of Management Reviews , 7 (2), 91-109.

CRAIG, D. (2013): «Los hoteles que aumentan su reputación online mejoran su ocupación e ingresos». Recuperado el 3 de Marzo de 2014, de Hosteltur: www.hosteltur. com/152800_hoteles-aumentan-su-reputacion-online-mejoran-su-ocupacion

CUENLLAS, A. (2012): «QQuién gestiona la reputación online de tu hotel?» Recuperado el 3 de Marzo de 2014, de Hosteltur: www.hosteltur.com/186160_quien-gestionareputacion-online-tu-hotel.html

CULLEN, K., \& HELSEL, C. (2010): The Evolving Dynamics of Revenue Management: a comprehensive revenue optimization road map for hotel owners, operators and practitioners. HSMAI Foundation.

CUNNINGHAM, P., SMYTH, B., WU, G., \& GREENE, D. (2010): «Does Tripadvisor Makes Hotels Better?» University College Dublin, School of Computer Science \& Informatics.

DE MAEYER, P. (2012): «Impact of online consumer reviews on sales and price strategies: a review and directions for future research». Journal of Product \& Brand Management, 132-139.

DEL FRESNO, M. (2012): El consumidor social: Reputación online y social media. Barcelona: UOC.

DELLAROCAS, C., FARAG AWAD, N., \& ZHANG, X. (2007): «Exploring the Value of Online Reviews to Organizations: Implications for Revenue Forecasting and Planning».

Estudio sobre Comercio Electrónico B2C 2012 (edición 2013). (2012): Recuperado el 26 de mayo de 2014, de www.ontsi.red.es: http://www.ontsi.red.es/ontsi/es/estudiosinformes/estudio-b2c-2012-edición-2013

FRIEDLANDER, R. (15 de febrero de 2013): «Como mejorar los ingresos con la reputación online». Recuperado el 30 de abril de 2013, de www.tecnohotelnews.com: http://www. tecnohotelnews.com/2013/02/como-mejorar-los-ingresos-con-la-reputacion-online/ 
FRIEDLANDER, R. (10 de Febrero de 2012): «Tendencias 2012 en la gestión de la reputación y las opiniones online de hoteles». Recuperado el 3 de Marzo de 2014, de www.reviewpro.com: http://www.reviewpro.com/es/tendencias-2012-en-la-gestion-dela-reputacion-y-las-opiniones-online-de-hoteles-6943

GRAHAM, M., \& BANSAL, P. (2007): «Consumers» Willingnes to Pay for Corporate Reputation: The Context of Airline Companies». Corporate Reputation Review , 10 (3), 189-200.

GRANDI BUSTILLOS, C. (2013): El uso de las redes sociales como estrategia de marketing en empresas del sector hostelero: una revisión del estado del arte. TFM, Universidad Politécnica de Valencia, Facultad de Administración y Dirección de Empresas.

GREEN, C., \& LOMANNO, M. (2012): Distribution Channel Analysis: a Guide for Hotels. HSMAI Foundation.

GRETZEL, U. (2007): Online Travel Review Study: Role \& Impact of Online Travel Reviews. Laboratory for Intelligent Systems in Tourism. Texas A\&M University.

GRETZEL, U., \& HYAN YOO, K. (2008): «Use and Impact of Online Travel Reviews» en Information and Communication Technologies. Proceedings of the International Conference (págs. 35-46). Innsbruck, Austria: Springer Vienna.

HELM, S. (2013): «How corporate reputation affects customers reactions to price increases». Journal of Revenue and Pricing Management , 12 (5), 402-415.

HERNÁNDEZ ESTÁRICO, E., FUENTES MEDINA, M., \& MORINI MARRERO, S. (2012): «Una aproximación a la reputación en línea de los establecimientos hoteleros españoles». Papers de Turisme (52), 63-88.

Holiday Hunting: Top 10 Travel sites in Europe. (24 de Abril de 2013): Recuperado el 25 de Marzo de 2014, de Comscore: www.comscoredatamine.com/2013/04/holidayhunting-top-10-travel-sites-in-europe/

HUBBS, J. (2014). «Social Media and its Growing Importance to Revenue Management». Recuperado el 3 de Marzo de 2014, de HSMAI: www.hsmai.org/knowledge

KAPLAN, A., \& HAENLEIN, M. (2010): «Users of the world, unite! The challenges and opportunities of Social Media». Business Horizons (53), 59-68.

KIMES, S.E. (1989): «The basics of yield management». Cornell Hotel and Restaurante Administration Quarterly 30(3), 14-19.

KOHLI, C., \& SURI, R. (2011): «The price is right? Guidelines for pricing to enhance profitability». Business Horizons (54), 563-573.

KOTLER, P., GARCÍA DE MADARIAGA MIRANDA, J., FLORES ZAMORA, J., BOWEN, J., \& MAKENS, J. (2011). Marketing Turístico. Madrid: Pearson Educación S.A.

LITVIN, S., GOLDSMITH, R., \& PAN, B. (2006). «Electronic Word-of-Mouth in Hospitality and Tourism Management». College of Charleston, School of Business and Economics.

LYNCH, J., \& ARIELY, D. (2000): «Wine Online: Search Costs Affect Competition on Price, Quality, and Distribution». Marketing Science , 19 (1), 83-103.

MACKENZIE, J. (1 de Agosto de 2012). «Revenue Management y Reputación Online». Recuperado el 3 de Marzo de 2014, de Hosteltur: www.hosteltur.com/198220_revenuemanagement-reputacion-online.html

MARTÍNEZ MARÍA-DOLORES, S., BERNAL GARCÍA, J., \& MELLINAS, J. (2012): «Los hoteles de la región de Murcia ante las redes sociales y la reputación online». Revista de Análisis Turístico (13), 1-10. 
MCCARTHY, L., STOCK, D., \& VERMA, R. (2010): «How Travelers Use Online and Social Media Channels to Make Hotel-Choice Decisions». Cornell Hospitality Report, 10 (18).

MELIÁN GONZÁLEZ, S., BULCHAND GIDUMAL, J., \& GONZÁLEZ LÓPEZ-VALCÁRCEL, B. (2010): «La participación de los clientes en sitios web de valoración de servicios turísticos. El caso de Tripadvisor». Revista de Análisis Turístico (10), 17-22.

MELLINAS CÁNOVAS, J. (2011): Redes Sociales y Turismo. Aproximación al caso de los hoteles de la Región de Murcia. TFM, Universidad Politécnica de Cartagena, Facultad de Ciencias de la Empresa.

MUÑOZ OÑATE, F. (1994): Marketing Turístico. Madrid: Centro de Estudios Ramón Areces, S.A.

NOONE, B. M., \& MCGUIRE, K. A. (2013): «Pricing in a social world: The influence of non-price information on hotel choice». Journal of Revenue and Pricing Management , $12(5), 385-401$.

NOONE, B. M., MCGUIRE, K. A., \& ROHLFS, K. V. (2011): «Social media meets hotel revenue management: Opportunities, issues and unanswered questions». Journal of Revenue and Pricing Management , 10 (4), 293-305.

NOONE, B., \& MCGUIRE, K. (Febrero de 2013): «Effects of Price and User Generated Content on Consumers Prepurchase Evaluations of Variablly Priced Services». Journal of Hospitality \& Tourism Research .

Online Consumer-generated Reviews have significant impact on offline purchase behaviour. (29 de Noviembre de 2007). Recuperado el 28 de Abril de 2014, de www. comscore.com: http://www.comscore.com/Insights/Press_Releases/2007/11/Online_ Consumer_Reviews_Impact_Offline_Purchasing_Behavior

PHILLIPS, R. (2005): Pricing and Revenue Optimization. California: Stanford University Press.

RESNICK, P., ZECKHAUSER, R., SWANSON, J., \& LOCKWOOD, K. (2006): «The value of reputation on Ebay: a controlled experiment». Experimental Economics , 9 (2), 79-101.

Revenue management and reviews: the perfect match for pricing it right. (26 de Febrero de 2013). Recuperado el 25 de Marzo de 2014, de EyeforTravel: eyefortravel.com/.../ revenue-managment-and-reviews-perfect-match-pricing-it-right

Review Sites: it's a regional thing. (4 de septiembre de 2013). Recuperado el 26 de mayo de 2014, de www.marketmetrix .com: http://corp.marketmetrix.com/review-sitesits-a-regional-thing/?utm_content=Patricia\&utm_source=VerticalResponse\&utm_ medium $=$ Email\&utm_term $=\& u t m \_c a m p a i g n=$ The $\% 20$ Breakdown $\% 3 \mathrm{~A} \% 20 \mathrm{Most} \% 20$ Influential\%20Review\%20Sites \%20by \%20Region\%2C\%20Complete\%20Customer\%20Feedback\%2C\%20and\%20Increasing\%20Player\%20Loyaltycontent

RODRÍGUEZ RUIBAL, A. (2010): «Utilización de las redes sociales como medio de promoción en el sector turístico. Opinión, valoración e interpretación de los comportamientos de los turistas». VIII Congreso «Turismo y Tecnologías de la Información y las Comunicaciones» Turitec.

ROSMAN, R., \& STUHURA, K. (2013): «The Implications of Social Media on Customer Relationship Management and the Hospitality Industry». Journal of Management Policy and Practice, 14 (3). 
SALVI, F., SERRA CANTALLOPS, A., \& RAMÓN CARDONA, J. (2013): «Los impactos del EWOM en hoteles». REDMARKA Revista Digital de Markting Aplicado, 2 (6), 3-17.

TripBarometer 2014 Global Report. (22 de abril de 2014). Recuperado el 27 de mayo de 2014, de www.tripadvisor.com: http://t4binsights-cache.tripadvisor.com/TripAdvisorInsights/sites/default/files/downloads/2200/tripbarometerglobalreport_us.pdf

Tripbarometer Winter 2012/2013. (9 de septiembre de 2013). Recuperado el 2014 de mayo de 2014, de www.tripadvisor.com: http://www.tripadvisor.com/TripAdvisorInsights/ n1914/tripbarometer-global

TUOMINEN, P. (2011): The Influence of TripAdvisor Consumer-Generated Travel Reviews on Hotel Performance. University of Hertfordshire.

VERMEULEN, I., \& SEEGERS, D. (2008): «Tried and tested: The impact of online hotel reviews on consumer consideration». Tourism Management , XXX, 1-5.

VINEREAN, S., CETINA, I., DUMITRESCU, L., \& TICHINDELEAN, M. (2013): «The Effects of Social Media Marketing on Online Consumer Behaviour». International Journal of Business and Management , 8 (14), 66-79.

WITHIAM, G. (2011): «Social Media and the Hospitality Industry: Holding the Tiger by the Tail». Cornell Hospitality Research Summit Proceedings, 3.

XIMÉNEZ DE SANDOVAL TORRES, J. (2013): Análisis cuantitativo de los hoteles en Tripadvisor: destinos costeros en España y Portugal. TFM, Universidad de Málaga, Facultad de Turismo.

YACOUEL, N., \& FLEISCHER, A. (2010): The Role of Cybermediaries in the Hotel Market. The Hebrew University of Jerusalem.

YE, Q., LAW, R., \& GU, B. (2009): «The impact of online user reviews on hotel room sales». International Journal of Hospitality Management (28), 180-182.

ZHU, F., \& ZHANG, X. (2010): «Impact of Online Consumer Reviews on Sales: The Moderating Role of Product and Consumer Characteristics». Journal of Marketing , 74, 133-148. 
